TP Periodica Polytechnica

Mechanical Engineering

61(4), pp. 303-308, 2017

https://doi.org/10.3311/PPme.11249

Creative Commons Attribution (i)

RESEARCH ARTICLE

\section{Friction Behavior of Engineering Polymers Treated by Atmospheric DBD Plasma}

\author{
Hayder Al-Maliki ${ }^{1 *}$, Gábor Kalácska ${ }^{1}$
}

Received 11 July 2017; accepted 14 September 2017

\begin{abstract}
The frictional behavior of (PA6 E and PETP) engineering polymers commonly used in the industry were investigated implying 3D surface topography due to Dielectric Barrier Discharge (DBD) source, atmospheric cold plasma surface treatment and compared to the pristine surface results under the same test conditions. The 3D surface topography shows a decrease in the surface roughness after treatment and keeps good topographical stability with the function of time. The friction coefficient of treated samples were lower than the pristine one under "runout" lubrication conditions in line with surface characterization results.
\end{abstract}

\section{Keywords}

friction, engineering polymers, atmospheric DBD plasma
${ }^{1}$ Ph.D. school of Mechanical Engineering,

Institute for Mechanical Engineering Technology, Szent István University, H-2100 Gödöllő, Páter Károly utca 1, Hungary

*Corresponding author, e-mail: haidrlatif@gmail.com

\section{Introduction}

Friction and wear are very important mechanisms that should be taken into consideration as well in the moving machine elements failure studies [1]. Engineering polymers widely used in moving part constructions especially in microelectronic, mechatronics and generally in industrial applications, due to its zero corrosion, lubricating ability, high strength, stiffness, and good dimensional stability; in contrast, they often experience difficulties in gluing [2], and instability or overload during sliding [3]. Some issues can be resolved by filling solid lubricants into the polymer [4], however, their initial bulk properties are also weak. According to above, surface modification comes to interest and what it can bring to light in further controlling the polymer performance simultaneously related to adhesion and tribology. Recently, among of different technologies of polymer surface treatment are a promising way to modify the tribological behavior as well. Amongst them, plasma is one of the most effective techniques at present [5] making it a valuable opportunity to investigate its effect on polymer surface for several studies last few years in terms of tribology and adhesion properties [6-8].

Previously studies have obviously shown the unpredictable effects of plasma surface modification on tribological behavior of polymers, due to the pluralism of governing mechanisms and parameters. It can be expected that functionalization, crosslinking, or chain scission will affect chemical and mechanical surface properties after plasma treatment, which in turn will alter the shear strength, friction, and wear. Where the tribological behavior of rubbers after plasma was accompanied improvements in friction and wear resistance [9]. Whereas higher friction was recorded for PETP after argon plasma treatment [10]. The scratch resistance of PETP indicates improvements due to the effect of nitrogen plasma immersion ion implantation (PIII) [11]. In a related study, it was demonstrated that the benefits on sliding tribological properties strongly depend on the sliding conditions: the lower friction and wear after PIII treatment only occurred at low pv conditions under dry or water-lubricated sliding and not at high pv conditions [6].

On the other hand, the tribological behavior of PA6 treated by N PIII plasma under different conditions (dry, water and oil 
lubrication) and certain pv regimes was studied [7]. It was concluded that under dry sliding conditions and low pv factor, the PA6 friction coefficient and specific wear are lower than the untreated one. Moreover, it was found that the water lubricant decreases the adhesive component of friction after treatment, whereas the oil lubricant decreases the sliding friction coefficient of PA6 as a consequence to increase of the dispersive component. Under continuous oil lubrication, no difference could be detected between the treated and untreated parameters. Whereas the runout condition was more efficient to detect this difference.

The literature viewing exposes the scarcity and the lack of the studies of polymer tribological behavior in term of plasma treatment, this was one of the motivations to delve into this topic.

The present paper deals with the friction behavior of polymer surface modified with dielectric barrier discharge (DBD) source atmospheric cold plasma process in polymer-metal contact-systems. The named process is suitable to modify efficiently the physical and chemical structures of polymer surfaces and thus change their surface characteristics including their tribological properties.

\section{Methods and materials \\ 2.1 Materials and preparations}

Two types of general engineering polymers have been investigated under commercial conditions (distributed by Quattroplast Ltd., Hungary and produced by Ensinger GmbH, Germany), were used in bulk conditions: Polyamide 6 extruded (PA6 E) grade Docamid-6-E (elastic modulus $\mathrm{E}=3300 \mathrm{MPa}$, tensile strength $\sigma=79 \mathrm{MPa}$, glass transition temperature $\mathrm{Tg}=45^{\circ} \mathrm{C}$ ), Polyethylene terephthalate (PETP) grade DocaPET (elastic modulus E $=3100 \mathrm{MPa}$, tensile strength $\sigma=79 \mathrm{MPa}$, glass transition temperature $\mathrm{Tg}=81^{\circ} \mathrm{C}$ ). Polymer samples were machined into pins with a diameter of $10 \mathrm{~mm}$ and thickness of $4 \mathrm{~mm}$. The surfaces were subsequently polished with wet $\mathrm{SiC}$ paper (grid numbers P1200 and P2000) and felt sheet towards required surface roughness. Before testing, the samples were thoroughly cleaned in an ultrasonic bath with distilled water and 96\% ethanol (Reanal, Hungary). The counterface of the tribological test was machined from the standard steel grade S235 (Ferroglobus Ltd., Hungary) The steel surfaces were ground and finally cleaned with Loctite SF 7063 (Henkel AG \& Co., Germany) according to the supplier's technology. The average roughness of the steel plates were $\mathrm{Ra}=0.72 \pm 0.02 \mu \mathrm{m}$ perpendicular to the sliding direction and $\mathrm{Ra}=0.46 \pm 0.02 \mu \mathrm{m}$ parallel to the sliding direction (measured with SurfTest SJ-201, Mitutoyo, Japan).

\subsection{Plasma treatment}

The atmospheric pressure ambient air plasma was generated by DCSBD plasma source at AKI, Research Centre for Natural Sciences. DCSBD plasma principally works depending on a coplanar DBD. The electrodes arranged in comb-shape within a dielectric. Two parallel strip-like electrode systems with standard dimensions of $1.5 \mathrm{~mm}$ wide, $\sim 0.5 \mathrm{~mm}$ thick, $1 \mathrm{~mm}$ strip to strip embedded in aluminum oxide matrix constitute the plasma panel. The DCSBD equipment was manufactured by Roplass s.r.o., Brno, Czech Republic. There is a ceramic layer between the plasma and electrodes has a $0.4 \mathrm{~mm}$ thickness. The DCSBD was designed to be suitable for flat surfaces treatment in particular. The DCSBD electrode system was powered by AC high voltage source which has a frequency $(10-20) \mathrm{kHz}$ and approximately $20 \mathrm{kV}$ peak-to-peak voltage. In the present study, the discharge power is $320 \mathrm{~W}$ and it was operated in the air in order obtain a quasi-homogeneous diffuse plasma surface. The system is equipped with oil cooling system in order to keep the system at the lowest possible temperature and maintain the gas temperature around $370 \mathrm{~K}$. The DCSBD plasma is described in detail [12]. The plasma treatment was performed in dynamic treatment mode and the distance between the treated polymer surface and DBD plate was $0.5 \mathrm{~mm}$. The treatment has been done under air atmosphere conditions $\left(\mathrm{T}=23^{\circ} \mathrm{C}, \mathrm{RH}=50 \%\right)$, the apparatus is shown in Fig. 1. The treatment time for each specimen was 1 min determined by preliminary experiments.

\section{Surface treatment by atmospheric cold plasma: dielectric barrier discharge (DBD)}

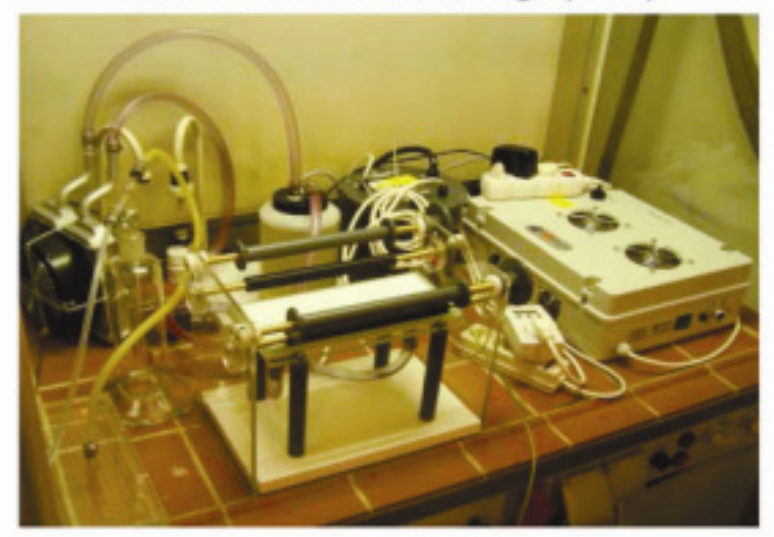

Fig. 1 DBD laboratory test equipment used for polymer surfaces

\subsection{D topography Surface characterization}

The surface roughness was evaluated from non-contact profilometry, using a 3D optical profilometer Coherence Correlation Interferometry (CCI) HD type (Taylor Hobson, Leicester, England) with an ultra-high precision closed loop piezoless z-scanner having a resolution in z-direction of $0.1 \AA$. Fiber Lite DC-950 source was used to generate a white light illumination and measurements were done at $50 \%$ light intensity. The elaborated surface area is $330 \times 330 \mu \mathrm{m} 2$ for each sample and it was imaged by vertical scanning interferometry has an objective lens with magnification $50 \mathrm{x}$ and numerical aperture $(\mathrm{NA})=0.55$. The scanning arrays contained 2048 x 2048 pixels with a field-of-view $(\mathrm{FOV})=330 \mu \mathrm{m}$, corresponding to a pixel size of $0.165 \mu \mathrm{m}$. The images were further processed by Talymap software (Digiserve) to calculate the $3 \mathrm{D}$ surface roughness parameters according to ISO 25178, including Sa (average roughness), Sz (maximum 
height), Sku (kurtosis) and Ssk (skewness). The roughness values were determined as average from three measurements at independent surface locations, with repeatability $\mathrm{Sa}<0.2 \AA$, more details about Coherence Scanning Interferometry in [13].

\subsection{Tribology test}

The tribological tests were carried out according to the VI. wear test category of the German standard DIN 50322 using a pin-on-disc test dynamic tribotester constructed at the Szent István University. Polymer pins with a diameter of $10 \mathrm{~mm}$ and height of $4 \mathrm{~mm}$ were mounted in a stationary holder and pressed against a rotating steel disc. During testing, the coefficient of friction $(\mu)$ was on-line monitored.

Based on our previous experiences "run-out" oil lubrication sliding test type was selected. The method is already introduced elsewhere [7]. The tests were performed using hydrophobic commercial gearbox oil (SAE 80W90) as a lubricant. $10 \mu \mathrm{l}$ oil was dropped onto the steel disc by a controlled pipette in front of the polymer contact zone before the first sliding period ( $0.5 \mathrm{~m}$ distance). In the second sliding period ( $9.5 \mathrm{~m}$ distance) the lubricant layer was removed by controlled wiping the sliding track on the steel surface with a labor wipe (Kimberly Clark). As such, (i) the first period of the sliding test corresponds to an oil-lubricating regime, while (ii) the second period of the sliding test can be considered as representative for a mixed or boundary lubrication regime, although the exact thickness of the lubricating oil film has not been further assessed due to its permanent change in thickness over time. The application of a thinned lubricating film was efficient to demonstrate differences in tribological properties between untreated and treated. The tests were run under a constant sliding velocity $\mathrm{v}=0.05 \mathrm{~m} / \mathrm{s}$, contact pressure $\mathrm{p}=0.5 \mathrm{MPa}$, and total sliding distance $10 \mathrm{~m}$.

\section{Results and discussion}

All the results were measured within 24 hours from plasma treatment time to prove fully surface modification obtaining and carried out at ambient conditions $\left(\mathrm{T}=23 \mathrm{C}^{\circ}, \mathrm{RH}=50 \%\right)$.

\subsection{Surface characterization}

Increase the surface wettability thereby surface energy of polymers due to atmospheric DBD plasma after short treatment time for PA6 and PET is reported in the literature $[14,15]$ also in our previous experience as well for PA6 [16]. This increase indicated to the presence of polar functionalities on the surface. The surface roughness is an important parameter, which influences the tribological behavior of the polymer [17]. Surface topography of pristine and plasma treated samples were monitored by non-contact profilometry, illustrated by 3D surface scans for PA6 E and PETP in Fig. 2. The 3D surface roughness parameters of pristine and treated surfaces are compared in Fig 3 . The initial Sa values of polymers are widely varied in spite of their similar preparation method before plasma treatment. Such difference in the roughness could be expected considering the dissimilar surface hardness. The influence of DBD plasma decreases the surface roughness where the average roughness Sa of PA6 E and PETP decreased $66 \%$ and $52 \%$ respectively. The decrease of the maximum height $(\mathrm{Sz})$ values suggests a greatly flattened surface probably owing to the removal of the top layer and melting of the surface asperities, while the original polishing grooves remain visible. In order to investigate the aging effect of plasma influence on polymer surface in term of topography, the tests were repeated after 800 hours. The polymers exhibit very good stability in surface topography. The surface smoothening is characteristic for the used plasma processing conditions and measurements at microlevel scale.

The common belief is that the DBD plasma can generally increase the surface roughness of polymers at nanoscale level due to etching effects after long times [18-20] whereas initial decreases in surface roughness might occur after shorter treatment times as other relative studies say it [15,21]. The nanoscale increase in the surface roughness is attributed to either polymer surface degradation or the constitution of nodule-link features or both of them. Nodule-links are composed by highly oxidized short polymer fragments, which called in the literature low molecular weight oxidized materials (LMWOMs) [19].

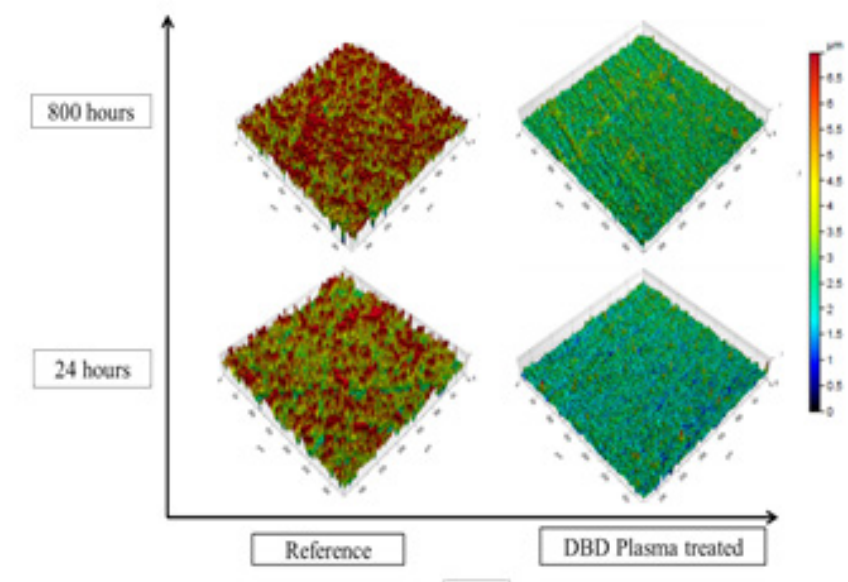

(a)

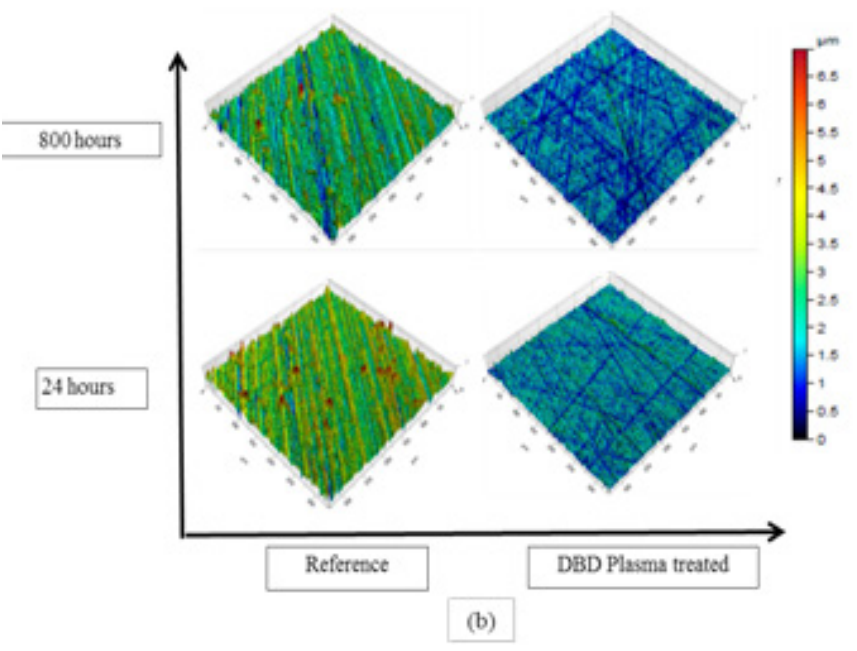

Fig. 2 Non-contact Profilometry of pristine and treated samples of (330 x $330 \mu \mathrm{m} 2$ surface area): (a) PA6 E and (b) PETP 


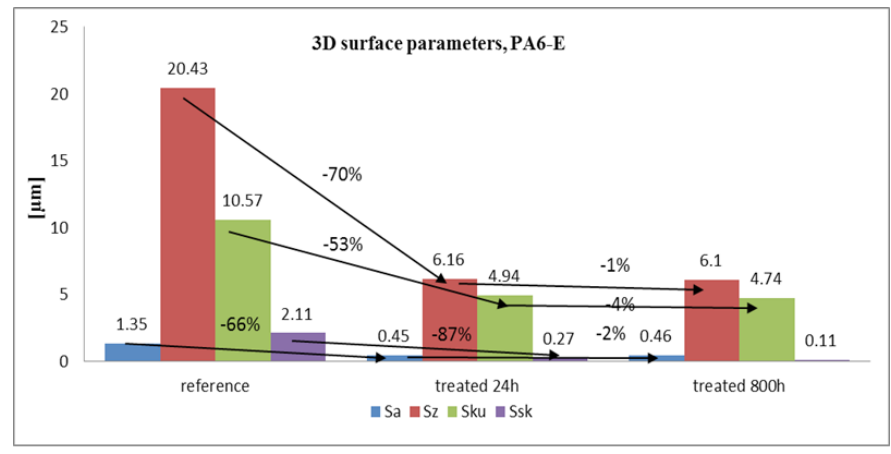

(a)

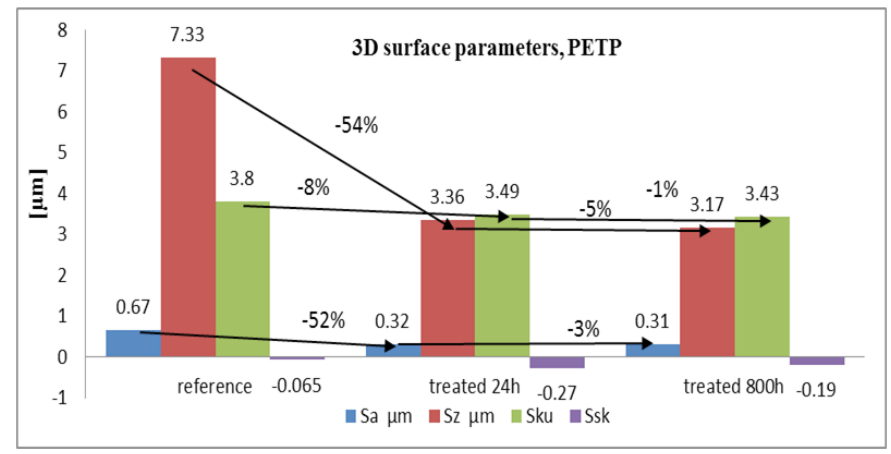

(b)

Fig. 3 3D surface roughness parameters of pristine compared to plasma treated samples after 24 hours and 800 hours: (a) PA6 E and (b) PETP

\subsection{Tribological tests}

The on-line measurements of friction coefficients of pristine and plasma treated samples under "run-out" oil lubricated sliding conditions are illustrated in Fig. 4. The comparative parameters of the maximum and average friction coefficients can be seen in Fig. 5 for polymers respectively. The friction coefficients in the early phase (first period) after application of an oil droplet are low $(\leq 0.05)$. No significant differences between the different samples (pristine and treated) could be observed due to the action of the oil film lubrication. In the beginning of sliding track cleaning remarkable and diversity behavior was noted between pristine and plasma treated samples, where plasma treated samples recorded lower friction coefficient compared to untreated one for both polymers.

The low friction of treated samples under "run-out" lubrication conditions can be attributed to enhance the oil retention capability in the sliding interface due to the surface microgeometrical modification in line with topography results and increase the surface energies in particular surface polarity, in accordance with former statement that surface energies of the plasma treated polymer surfaces are significantly higher than the pristine surfaces [16]. A combined effect of the mentioned factors could lead to affirmative improvement in the adsorption of the hydrophobic oil lubricant on the polymer surface and entrapment in the interface.

From friction behavior which monitored in Fig. 4 obviously could be seen the periodic fluctuations in friction during the phase of removing the oil from the sliding track. The optimal

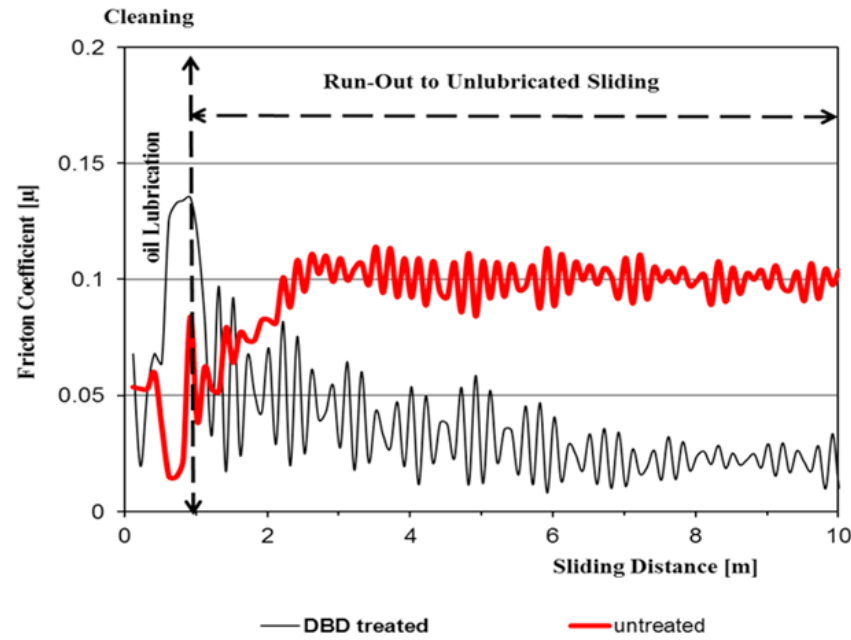

(a)

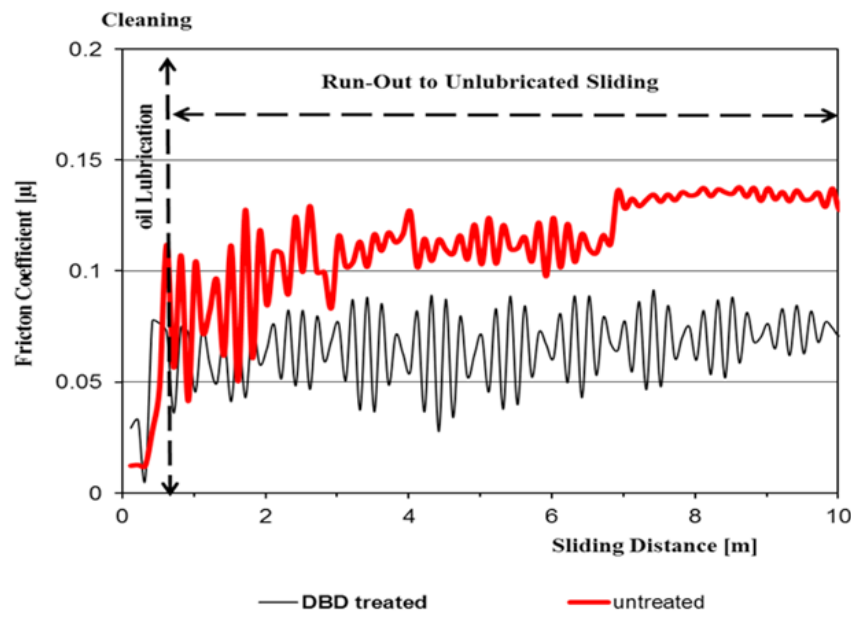

(b)

Fig. 4 Friction behavior under lubricated sliding and run-out conditions at 0.5 MPa: (a) PA6 E and (b) PETP

interpretation for this is the friction instabilities within the mixed lubrication regime due to the dynamic changes in lubricant layer thickness, where the lubricant is removed from the contact area. The remaining lubricant after decreasing thickness layer is sufficient to keep the friction stable at a lower level compared to the pristine sample in spite of the same test conditions were applied for both samples.

PETP observed the higher friction coefficient for pristine surface compared to PA6 E, which may attribute to the influence of mechanical properties (hardness, strength, and stiffness) and surface energies.

In general, the average of friction coefficient after removing the lubricant from the sliding track (steady state) for PA6 E and PETP decreased $230 \%$ and $80 \%$ respectively due to atmospheric DBD plasma treatment. These results were in line with the surface characterization results, where PA6 E has the higher polar component of surface energy [16] and surface 


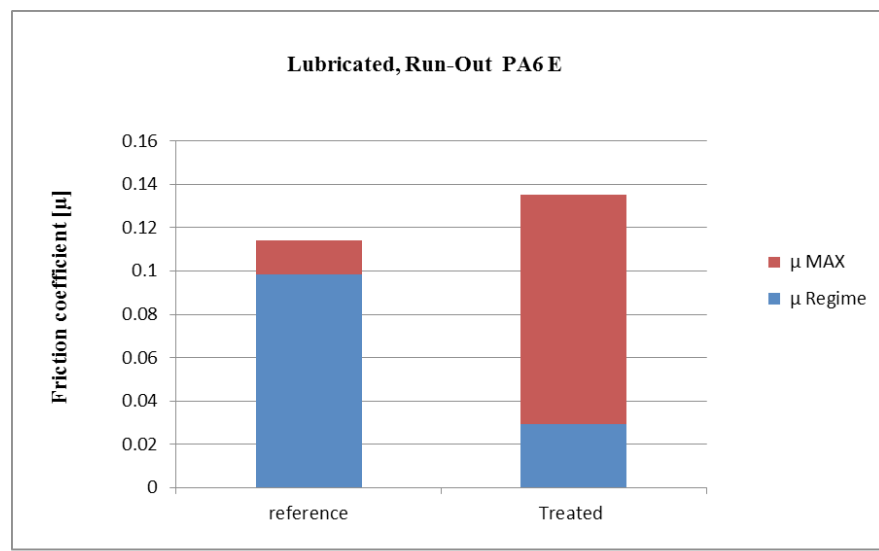

(a)

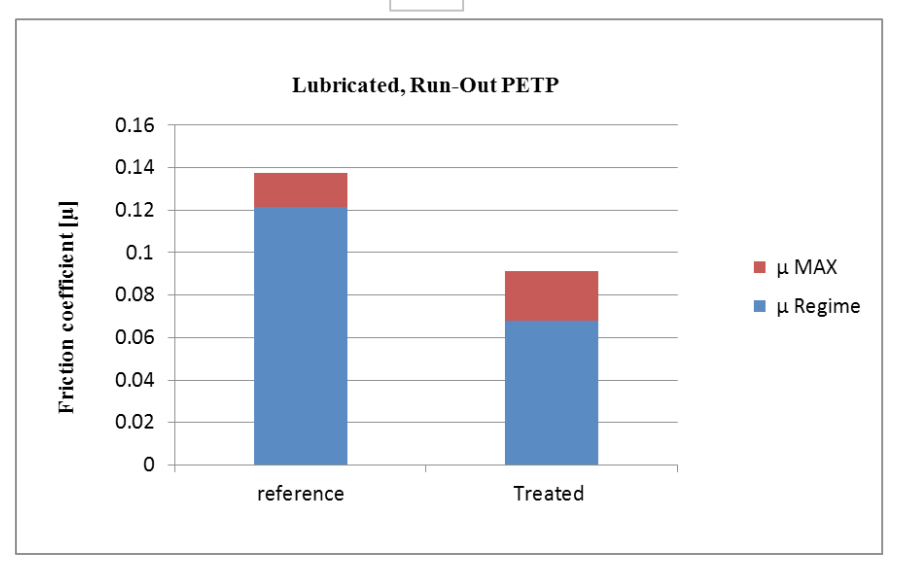

(b)

Fig. 5 Averages and maximum friction coefficients: (a) PA6 E and (b) PETP

mean roughness (smaller real contact area) although obtained stronger decrease of friction after treatment, thereby PA6 E offered better lubrication performance.

\section{Conclusion}

The bottom line of this paper is that atmospheric DBD plasma can be an effective technique to improve the friction behavior of engineering polymers under mild conditions. Further research is going to clarify the validity of this statement for other materials under various conditions.

Two widely used engineering polymers PA6 E and PETP have been examined after and before atmospheric DBD plasma treatment from the point of oil lubricated sliding on steel surface. The surface topography was investigated by using 3D optical profilometer Coherence Correlation Interferometry (CCI) HD type. The pin-on-disc test was selected to examine the friction behavior under "run-out" oil lubrication conditions. The results exhibited that the surface roughness of both polymers were decreased upon plasma treatment and show good stability with the time function. The friction coefficient of treated polymer surfaces were lower than the virgin one for longer sliding time due to the surface microgeometrical modification and enhanced oil retention capability after raising the surface energy upon plasma treatment, which improved the oil lubrication performance.

\section{Acknowledgement}

The research is supported by OTKA K 113039 and 3D topographical investigation was carried out in co-operation with Laboratory Soete, Gent University, Belgium. DBD plasma treatment was carried out at AKI, Research Centre for Natural Sciences, Hungarian Academy of Sciences.

\section{References}

[1] Zsidái, L., De Baets, P., Samyn, P., Kalácska, G., Van Peteghem, A. P., Van Parys, F. "The tribological behaviour of engineering plastics during sliding friction investigated with small-scale specimensle." Wear. 253, pp. 673-688. 2002.

https://doi.org/10.1016/S0043-1648(02)00149-7

[2] Pandiyaraj, K. N., Selvarajan, V., Deshmukh, R. R., Gao. C. "Adhesive properties of polypropylene (PP) and polyethylene terephthalate (PET) film surfaces treated by DC glow discharge plasma." Vacuum. 83(2), pp. 332-339. 2008.

https://doi.org/10.1016/j.vacuum.2008.05.032

[3] Samyn, P., Quintelier, J., Ost, W., De Baets, P., Schoukens, S. "Sliding behaviour of pure polyester and polyester-PTFE filled bulk composites in overload conditions." Polymer Testing. 24(5), pp. 588-603. 2005. https://doi.org/10.1016/j.polymertesting.2005.02.012

[4] Samyn, P., Van Craenenbroeck, J., Quintelier, J., Schoukens, G. "Friction induced conformational changes on large-scale and small-scale polyester sliding surfaces." Surface and Interface Analysis. 38(4), pp. 868-872. 2006. https://doi.org/10.1002/sia.2161

[5] Chan, C-M., Ko, T-M., Hiraoka , H. "Polymer surface modification by plasmas and photons." Surface Science Reports. 24(1-2), pp. 1-54. 1996. https://doi.org/10.1016/0167-5729(96)80003-3

[6] Kalácska, G., Zsidai, L., Kereszturi, K., Mohai, M., Tóth, A. "Sliding tribological properties of untreated and PIII-treated PETP." Applied Surface Science. 255(11), pp. 5847-5850. 2009. https://doi.org/10.1016/j.apsusc.2009.01.017

[7] Kalácska, G., Zsidai, L., Keresztes, R., Tóth, A., Mohai, Szépvölgyi, J. "Effect of nitrogen plasma immersion ion implantation of polyamide- 6 on its sliding properties against steel surface." Wear. 290-291, pp. 66-73. 2012. https://doi.org/10.1016/j.wear.2012.05.011

[8] Abdul Samad, M., Satyanarayana, M., Sinha, K. S. "Tribology of UHMWPE film on air-plasma treated tool steel and the effect of PFPE overcoat." Surface \& Coatings Technology. 204(9-10), pp. 1330-1338.2010. https://doi.org/10.1016/j.surfcoat.2009.09.011

[9] Segu, D. Z. "NBR surface modification by Ar plasma and its tribological properties." Industrial Lubrication and Tribology. 68(2), pp. 227 232. 2016.

https://doi.org/10.1108/ILT-05-2015-0062

[10] Beake, G. J., Leggett, B. D. "Development of surface morphology, local friction and adhesion in plasma-treated poly(ethylene terephthalate) films." American Chemical Society, Polymer Preprints. 39(2), pp. 12281229. 1998.

[11] Kereszturi, K., Tóth, A., Mohai, M., Bertóti, I. "Surface chemical and nanomechanical alterations in plasma immersion ion implanted PET." Surface and Interface Analysis. 40(3-4), pp. 664-667. 2008. https://doi.org/10.1002/sia.2643

[12] Černák, M., Černáková, L., Hudec, I., Kováčik, D., Zahoranová, A. "Diffuse Coplanar Surface Barrier Discharge and its applications for in-line processing of low-added-value materials." European Physical Journal: Applied Physics. 47, pp. 22806. 2009.

[13] De Groot, P. "Coherence Scanning Interferometry." In: Optical Measurement of Surface Topography. (pp. 187-208). Springer, Berlin. 2011. https://doi.org/10.1007/978-3-642-12012-1 
[14] Kostov, K. G., Hamia, Y. A. A., Mota, R. P., dos Santos, A. I. R., Nascente, P. A. P. "Treatment of polycarbonate by dielectric barrier discharge (DBD) at atmospheric pressure." Journal of Physics: Conference Series. 511(1), pp. 12075. 2014. [Online]. Available from: http://iopscience.iop.org/article/10.1088/1742-6596/511/1/012075 [Accessed: 11th July 2017]

[15] Novák, I., Popelka, A., Valentín, M., Chodák, I., Špírková, M., Tóth, A., Kleinová, A., Sedliacik, J., Lehocký, M., Marônek, M. "Surface Behavior of Polyamide 6 Modified by Barrier Plasma in Oxygen and Nitrogen." International Journal of Polymer Analysis and Characterization. 19(1), pp. 31-38. 2014.

https://doi.org/10.1080/1023666X.2014.850907

[16] Károly, Z., Klébert, Sz., Al-Maliki, H., Pataki, T. "Comparison of NPIII and DBD Plasma Treatment in Terms of Wettability of PTFE and PA6." SCIENTIFIC BULLETIN, Serie C, Fascicle: Mechanics, Tribology, Machine Manufacturing Technology. 2016(xxx), pp. 165-170. 2016. [Online]. Available from: http://real.mtak.hu/id/eprint/45478 [Accessed: 11th July 2017]

[17] Kumar, N. N., Yap, S. L., bt Samsudin Srinivasa, F. N. D., Khan, M. Z., Srinivasa, R. S. P. "Effect of Argon Plasma Treatment on Tribological Properties of UHMWPE/MWCNT Nanocomposites." Polymers. 8(8), pp. 295. 2016.

https://doi.org/10.3390/polym8080295
[18] Ionita, M. D., Teodorescu, M., Stancu, C., Stancu, E. C., Ionita, E. R., Moldovan, Acsente, M. B., Dinescu, G. "Surface modification of polymers at atmospheric pressure in expanding RF plasmas generated by planar dielectric barrier discharges." Journal of Optoelectronics and Advanced Materials. 12(3), 2010. [Online]. Available from: https://joam. inoe.ro/index.php?option $=$ magazine $\&$ op $=v i e w \& i d u=2407 \&$ catid $=48$ [Accessed: 11th July 2017]

[19] Kostov, K. G., Nishime, T. M. C, Hein, L. R. O., Toth, A. "Study of polypropylene surface modification by air dielectric barrier discharge operated at two different frequencies." Surface and Coatings Technology. 234, pp. 60-66. 2013.

https://doi.org/10.1016/j.surfcoat.2012.09.041

[20] Nastuta, A. V., Rusu, G. B., Topala, I., Chiper, A. S., Popa, G. "Surface modifications of polymer induced by atmospheric DBD plasma in different configurations." Journal of Optoelectronics and Advanced Materials. 10(8), pp. 2038-2042. 2008. Retrieved from https:/joam. inoe.ro/index.php?option $=$ magazine $\&$ op $=$ view $\&$ idu $=1548 \&$ catid $=29$ [Accessed: 11th July 2017]

[21] Hergelova, B., Homola, T., Zahoranava, A., Plecenik, T., Kovacik, D., Matousek, J. "Plasma surface modification of biocompatible polymers using atmospheric pressure dielectric barrier discharge." In: WDS Conference proceedings. (pp. 128-133). Praha. 2012. [Online]. Available from: https://www.muni.cz/vyzkum/publikace/1083764 [Accessed: 11th July 2017] 